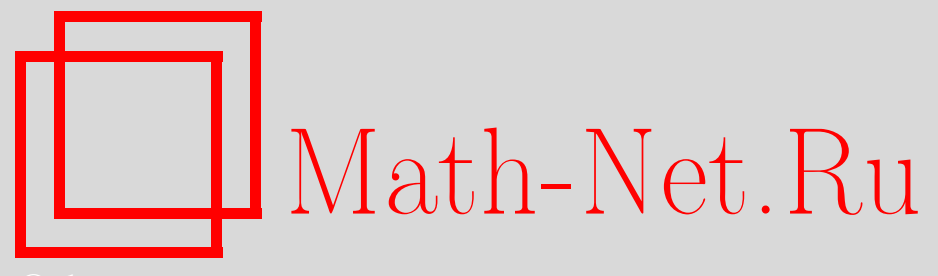

В. П. Елизаров, Разрешимые и локально замкнутые модули и кольца, Дискрет. матем., 2006, том 18, выпуск 1, 3039

DOI: https://doi.org/10.4213/dm30

Использование Общероссийского математического портала Math-Net.Ru подразумевает, что вы прочитали и согласны с пользовательским соглашением http://www.mathnet.ru/rus/agreement

Параметры загрузки:

IP : 3.85 .5 .30

26 апреля 2023 г., 13:57:38

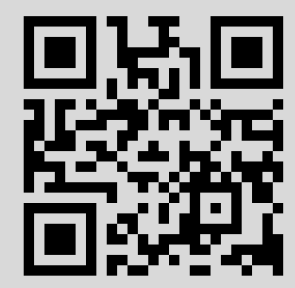




\title{
Разрешимые и локально замкнутые модули и кольца
}

\author{
๑) 2006 г. В. П. Елизаров
}

Описаны классы всех модулей и всех колец, системы линейных уравнений над которыми разрешимы тогда и только тогда, когда они согласованы относительно линейных соотношений.

Работа выполнена при поддержке программы Президента Российской Федерации поддержки ведущих научных школ, грант НШ 2358.2003.9.

\section{1. Введение}

Термин кольцо далее означает ассоциативное кольцо с единицей, а термин модуль унитарный модуль. Запись $T_{R}$ или ${ }_{R} T$ означает, что $T$ - правый или соответственно левый модуль над кольцом $R$. Для множества $F$ через $F_{m, n}$ обозначим совокупность всех $m \times n$ матриц с элементами из $F$. В частных случаях полагаем

$$
F_{1, n}=F(n), \quad F_{m, 1}=F^{(m)} .
$$

Рассматриваются системы левых линейных уравнений над модулем ${ }_{R} \Lambda$, то есть системы уравнений вида

$$
A \xi^{\downarrow}=\beta^{\downarrow}
$$

где $A \in R_{m, n}, \beta^{\downarrow} \in \Lambda^{(m)}$ и $\xi^{\downarrow}-$ вектор неизвестных со значениями из $\Lambda^{(n)}$. Система уравнений (1) называется разрешимой, если она имеет хотя бы одно решение.

Через 0 и $\theta$ обозначим нули соответственно кольца $R$ и модуля $\Lambda$. Система уравнений (1) называется согласованной относительно линейных соотношений (lc-согласованной), если для любого вектора $\vec{C} \in R(m)$ справедлива импликация

$$
\vec{C} A=\overrightarrow{0} \quad \Longrightarrow \quad \vec{C} \beta^{\downarrow}=\theta
$$

(при $\Lambda=R$ этот термин введен в [2]).

Очевидно, что разрешимая система уравнений (1) является $l c$-согласованной. Обратное в общем случае неверно. Простейший пример - уравнение $2 x_{1}=1$ над модулем $\mathbf{Z}$, где $\mathbf{Z}$ - кольцо целых чисел.

При $\Lambda=R$ систему уравнений (1) запишем в виде

$$
A X^{\downarrow}=B^{\downarrow}
$$


где $B^{\downarrow} \in R^{(m)}$ и $X^{\downarrow}$ - вектор неизвестных со значениями из $R^{(n)}$. Известны некоторые классы колец, для системы уравнений вида (2) над которыми критерий разрешимости $l c$-согласованность: тела (см. §105 в [10]), коммутативные цепные кольца, над которыми разрешимо любое $l c$-согласованное уравнение вида

$$
a x_{1}=b
$$

(см. [7]), конечные коммутативные квазифробениусовы кольца (см. [5, 6]), произвольные квазифробениусовы кольца (см. [1]).

В [1] была сформулирована задача описать класс всех колец, над которыми разрешима любая $l c$-согласованная система уравнений вида (2). В [2] такие кольца названы $l c$-совместными, а в [3] - $l c$-разрешимыми. Указанная задача является частью Проблемы 2 из [3]. В [1] задача была решена в классе $\mathcal{N}$ нетеровых слева и справа колец: класс всех $l c$-разрешимых колец из $\mathcal{N}$ - это в точности класс квазифробениусовых колец.

В настоящей работе решается задача более общая, чем задача, поставленная в [1], и Проблема 2 из [3]. А именно, в $§ 2$ описан класс $\mathscr{K}(R)$ всех $R$-модулей, для систем уравнений вида (1) над которыми критерием разрешимости является $l c$-согласованность. Описание внутреннее, то есть использует только свойства самих модулей. Как следствие получается описание класса всех $l c$-разрешимых колец. В 33 указан один подкласс класса $\mathscr{K}(R)$ и, как следствие, один подкласс класса $l c$-разрешимых колец, в котором содержится класс квазифробениусовых колец.

\section{2. Класс $l c$-разрешимых модулей}

Введем некоторые понятия и обозначения.

Определение 1. Модуль ${ }_{R} \Lambda$ назовем $l c$-разрешимым, если любая $l c$-согласованная система уравнений вида (1) является разрешимой.

Для подмножеств $S \subset R^{(m)}, T \subset R(m)$ и вектора $\gamma^{\downarrow} \in \Lambda^{(m)}$ определим аннуляторы в $R(m)$ и $\Lambda^{(m)}$, полагая

$$
\begin{aligned}
\operatorname{Ann}_{R(m)} S & =\{\vec{C} \in R(m) \mid \vec{C} S=\{0\}\}, \\
\operatorname{Ann}_{R(m)} \gamma^{\downarrow} & =\left\{\vec{D} \in R(m) \mid \vec{D} \gamma^{\downarrow}=\theta\right\}, \\
\operatorname{Ann}_{\Lambda^{(m)}} T & =\left\{\delta^{\downarrow} \in \Lambda^{(m)} \mid T \delta^{\downarrow}=\{\theta\}\right\} .
\end{aligned}
$$

Введем также обозначение

$$
S \Lambda=\left\{\sum_{i=1}^{k} S_{i}^{\downarrow} \lambda_{i} \mid k \in \mathbf{N}, S_{i}^{\downarrow} \in S, \lambda_{i} \in \Lambda\right\}
$$

Определение 2. Модуль ${ }_{R} \Lambda$ назовем локально $R$-замкнутым справа, если для любого $m \in \mathbf{N}$ и для любого конечно порожденного подмодуля $L$ из $R_{R}^{(m)}$ справедливо равенство

$$
\operatorname{Ann}_{\Lambda}^{(m)} \operatorname{Ann}_{R(m)} L=L \Lambda .
$$

Для удобства ссылок сформулируем два утверждения. 
Предложение 1. Для $S \subset R^{(m)} u \gamma^{\downarrow} \in \Lambda^{(m)}$ справедливы соотношения

$$
\begin{gathered}
\operatorname{Ann}_{\Lambda^{(m)}} \operatorname{Ann}_{R(m)} S \supset S \Lambda, \\
\operatorname{Ann}_{\Lambda^{(m)}} \operatorname{Ann}_{R(m)} \gamma^{\downarrow} \ni \gamma^{\downarrow} .
\end{gathered}
$$

Ввиду предложения 1 определение 2 корректно.

Для векторов $D_{1}^{\downarrow}, \ldots, D_{k}^{\downarrow}$ из $R^{(m)}$ обозначим через $\left\langle D_{1}^{\downarrow}, \ldots, D_{k}^{\downarrow}\right\rangle$ порожденный ими подмодуль модуля $R_{R}^{(m)}$.

Предложение 2. Справедливо равенство

$$
\operatorname{Ann}_{R(m)}\left\{D_{1}^{\downarrow}, \ldots, D_{k}^{\downarrow}\right\}=\operatorname{Ann}_{R(m)}\left\langle D_{1}^{\downarrow}, \ldots, D_{k}^{\downarrow}\right\rangle .
$$

Получим критерий разрешимости системы уравнений (1) над локально $R$-замкнутым справа модулем ${ }_{R} \Lambda$.

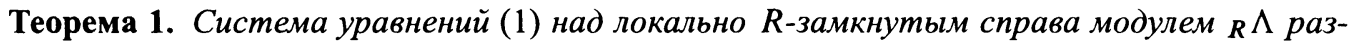
решима тогда и только тогда, когда она lс-согласованная.

Доказательство. Докажем только нетривиальную часть теоремы. Пусть система уравнений (1) lc-согласованная,

$$
\begin{aligned}
A & =\left(A_{1}^{\downarrow}, \ldots, A_{n}^{\downarrow}\right), \\
M & =\left\langle A_{1}^{\downarrow}, \ldots, A_{n}^{\downarrow}\right\rangle .
\end{aligned}
$$

Условие $l c$-согласованности можно записать в виде

$$
\operatorname{Ann}_{R(m)}\left\{A_{1}^{\downarrow}, \ldots, A_{n}^{\downarrow}\right\} \subset \operatorname{Ann}_{R(m)} \beta^{\downarrow}
$$

или, согласно предложению 2 , в виде

$$
\operatorname{Ann}_{R(m)} M \subset \operatorname{Ann}_{R(m)} \beta^{\downarrow} .
$$

Из (4) следует соотношение

$$
\mathrm{Ann}_{\Lambda^{(m)}} \operatorname{Ann}_{R(m)} M \supset \mathrm{Ann}_{\Lambda^{(m)}} \mathrm{Ann}_{R(m)} \beta^{\downarrow}
$$

По условию и предложению 1 из (5) получаем, что

$$
M \Lambda \ni \beta^{\downarrow} \text {. }
$$

Стало быть, справедливо равенство

$$
\beta=\sum_{i=1}^{k}\left(\sum_{j=1}^{n} A_{j}^{\downarrow} d_{j i}\right) \lambda_{i},
$$

где $k \in \mathbf{N}, d_{i j} \in R$ и $\lambda_{i} \in \Lambda$. Равенство (6) перепишем в виде

$$
\beta^{\downarrow}=\sum_{j=1}^{n} A_{j}^{\downarrow} \mu_{j},
$$


где

$$
\mu_{j}=\sum_{i=1}^{k} d_{j i} \lambda_{i}, \quad j \in\{1, \ldots, n\}
$$

Таким образом, вектор

$$
\mu^{\downarrow}=\left(\mu_{1}, \ldots, \mu_{n}\right)^{T}
$$

является решением системы уравнений (1).

Следующее утверждение показывает, что условие на модуль ${ }_{R} \Lambda$ в теореме 1 существенно.

Предложение 3. Если модуль ${ }_{R} \Lambda$ не является локально $R$-замкнутым справа, то существует lс-согласованная система левых линейных уравнений над ${ }_{R} \Lambda$, не имеющая решений.

Доказательство. Ввиду условия, определения 2 и предложения 1 найдутся такие $t \in \mathbf{N}$ и подмодуль $H=\left\langle D_{1}^{\downarrow}, \ldots, D_{p}^{\downarrow}\right\rangle$ модуля $R_{R}^{(t)}$, что

$$
\operatorname{Ann}_{\Lambda}^{(t)} \operatorname{Ann}_{R(t)} H \supsetneqq H \Lambda .
$$

Поэтому существует такой вектор $\alpha^{\downarrow} \in \Lambda^{(t)}$, что справедливы соотношения

$$
\operatorname{Ann}_{R(t)} H \alpha^{\downarrow}=\{\theta\}
$$

и

$$
\alpha^{\downarrow} \notin H \Lambda \text {. }
$$

Рассмотрим систему уравнений

$$
D \xi^{\downarrow}=\alpha^{\downarrow}
$$

над модулем ${ }_{R} \Lambda$, где

$$
D=\left(D_{1}^{\downarrow}, \ldots, D_{p}^{\downarrow}\right) \in R_{t, p} .
$$

Ввиду (7) и предложения 2 система уравнений (9) $l c$-согласованная, но соотношение (8) означает, что у нее нет решений.

Теорема 1 и предложение 3 позволяют внутренним образом охарактеризовать класс $l c$-разрешимых $R$-модулей.

Tеорема 2. Класс lс-разрешимых $R$-модулей - это в точности класс локально $R$-замкнутых справа модулей.

Класс всех локально $R$-замкнутых справа модулей обозначим через $\mathscr{K}(R)$.

Применим полученные результаты в случае, когда $\Lambda=R$.

Определение 3. Кольцо $R$ называется $l c$-разрешимым, если любая $l c$-согласованная система уравнений над $R$ вида (2) разрешима [2, 3].

Определение 4. Кольцо $R$ назовем локально замкнутым справа, если для любого $m \in \mathbf{N}$ и любого конечно порожденного подмодуля $L$ из $R_{R}^{(m)}$ справедливо равенство

$$
\operatorname{Ann}_{R(m)} \operatorname{Ann}_{R(m)} L=L .
$$


Проводя рассуждения, аналогичные проведенным при доказательстве теоремы 1 и предложения 3, получаем внутреннее описание класса всех $l c$-разрешимых колец.

Теорема 3. Класс lс-разрешимых колеч - это в точности класс локально замкнутых справа колеч.

\section{3. Подкласс класса $\mathscr{K}(R)$}

В этом параграфе мы укажем некоторый подкласс класса модулей $\mathscr{K}(R)$ и некоторый подкласс класса локально замкнутых справа колец.

Определение 5. Модуль $R \Lambda$ назовем замкнутым относительно конечно порожденных правых идеалов кольца $R$ (или, коротко, $\alpha$-модулем), если для любого конечно порожденного правого идеала $J$ кольца $R$ справедливо равенство

$$
\operatorname{Ann}_{\Lambda} \operatorname{Ann}_{R} J=J \Lambda
$$

По определению 2 всякий локально $R$-замкнутый справа модуль ${ }_{R} \Lambda$ является $\alpha$-модулем.

Предложение 4. Уравнение

$$
\vec{A} \xi^{\downarrow}=\beta
$$

над $\alpha$-модулем ${ }_{R} \Lambda$, где $\vec{A} \in R(n) u \beta \in \Lambda$, разрешимо тогда и только тогда, когда оно lс-согласовано.

Это утверждение является частным случаем теоремы 1.

Лемма 1 ([2]). Если система уравнений (1) lc-согласованная, то lс-согласована и любая ее подсистема.

Предложение 5. Если $n \in \mathbf{N} u T, F-$ подмножества $R(n)$, то при

$$
T \cap F=\varnothing
$$

справедливо включение

$$
\operatorname{Ann}_{\Lambda^{(n)}}(T \cap F) \supset \operatorname{Ann}_{\Lambda^{(n)}} T+\operatorname{Ann}_{\Lambda^{(n)}} F
$$

Доказательство стандартно.

Определение 6. Модуль ${ }_{R} \Lambda$ назовем $\delta$-модулем, если для любого $n \in \mathbf{N}$ и для любых конечно порожденных подмодулей $K$ и $L$ из ${ }_{R} R(n)$ справедливо равенство

$$
\operatorname{Ann}_{\Lambda^{(n)}}(K \cap L)=\operatorname{Ann}_{\Lambda^{(n)}} K+\operatorname{Ann}_{\Lambda^{(n)}} L
$$

Ввиду предложения 5 определение 6 корректно.

Теорема 4. Система уравнений (1) над $\alpha$ - $и \delta$-модулем ${ }_{R} \Lambda$ разрешима тогда и только тогда, когда она lс-согласована. 
Доказательство. Докажем только нетривиальную часть теоремы. Пусть система уравнений (1) $l c$-согласованная.

При $m=1$ система уравнений (1) разрешима по предложению 4.

Пусть $m=2$. По лемме 1 каждое уравнение системы (1) $l c$-согласовано, и по случаю $m=1$ оно разрешимо. Значит, при некоторых векторах $\mu_{i}^{\downarrow} \in \Lambda^{(n)}$ справедливы равенства

$$
\vec{A}_{i} \mu_{i}^{\downarrow}=\beta_{i}, \quad i=1,2 \text {. }
$$

Через $\left\langle\vec{A}_{i}\right\rangle$ обозначим подмодуль из $R R(n)$, порожденный вектором $\vec{A}_{i}$. Так как справедливо равенство

$$
\operatorname{Ann}_{\Lambda^{(n)}} \vec{A}_{i}=\operatorname{Ann}_{\Lambda^{(n)}}\left\langle\vec{A}_{i}\right\rangle, \quad i=1,2,
$$

то множество $\Omega_{i}$ всех решений $i$-го уравнения системы (1) записывается в виде

$$
\Omega_{i}=\mu_{i}^{\downarrow}+\operatorname{Ann}_{\Lambda^{(n)}}\left\langle\vec{A}_{i}\right\rangle, \quad i=1,2 .
$$

Покажем, что

$$
\Omega_{1} \cap \Omega_{2} \neq \varnothing
$$

Пусть

$$
\vec{U} \in\left\langle\vec{A}_{1}\right\rangle \cap\left\langle\vec{A}_{2}\right\rangle
$$

то есть

$$
\vec{U}=c_{1} \vec{A}_{1}=c_{2} \vec{A}_{2}
$$

при некоторых $c_{1}, c_{2} \in R$. Из равенства

$$
\left(c_{1},-c_{2}\right)\left(\begin{array}{l}
\vec{A}_{1} \\
\vec{A}_{2}
\end{array}\right)=\overrightarrow{0}
$$

ввиду $l c$-согласованности системы уравнений (1) следует равенство

$$
\left(c_{1},-c_{2}\right)\left(\begin{array}{l}
\beta_{1} \\
\beta_{2}
\end{array}\right)=\theta
$$

то есть равенство

$$
c_{1} \vec{A}_{1} \mu_{1}^{\downarrow}+\left(-c_{2}\right) \vec{A}_{2} \mu_{2}^{\downarrow}=\theta
$$

или равенство

$$
\vec{U}\left(\mu_{1}^{\downarrow}-\mu_{2}^{\downarrow}\right)=\theta .
$$

Таким образом, справедливо соотношение

$$
\mu_{1}^{\downarrow}-\mu_{2}^{\downarrow} \in \operatorname{Ann}_{\Lambda^{(n)}}\left(\left\langle\vec{A}_{1}\right\rangle \cap\left\langle\vec{A}_{2}\right\rangle\right) .
$$

Так как ${ }_{R} \Lambda-\beta$-модуль, из (10) получаем, что

$$
\mu_{1}^{\downarrow}-\mu_{2}^{\downarrow} \in \operatorname{Ann}_{\Lambda^{(n)}}\left\langle\vec{A}_{1}\right\rangle+\operatorname{Ann}_{\Lambda^{(n)}}\left\langle\vec{A}_{2}\right\rangle .
$$

Поэтому существуют такие векторы $\delta_{i}^{\downarrow} \in \operatorname{Ann}_{\Lambda^{(n)}}\left\langle\vec{A}_{i}\right\rangle, i \in\{1,2\}$, что

$$
\mu_{1}^{\downarrow}+\delta_{1}^{\downarrow}=\mu_{2}^{\downarrow}+\delta_{2}^{\downarrow} \in \Omega_{1} \cap \Omega_{2} \text {. }
$$


Это означает, что при $m=2$ система уравнений (1) разрешима.

Предположим, что при произвольном фиксированном $k \geqslant 2$ любая $l c$-согласованная система левых линейных уравнений над ${ }_{R} \Lambda$, состоящая из $k$ уравнений от $n$ неизвестных, разрешима, и пусть система (1) содержігт $m=k+1$ уравнений.

По лемме $1 l c$-согласованы подсистемы системы уравнений (1)

$$
\vec{A} \xi^{\downarrow}=\beta^{\downarrow}
$$

и

$$
\left(\begin{array}{c}
\vec{A}_{1} \\
\vec{A}_{k+1}
\end{array}\right) \xi^{\downarrow}=\left(\begin{array}{c}
\beta_{1} \\
\beta_{k+1}
\end{array}\right)
$$

состоящие соответственно из первых $k$ уравнений и из 1-го и $k+1$-го уравнений.

По предположению индукции и случаю $m=2$ системы уравнений (11) и (12) разрешимы. Запишем множества $\Sigma_{1}$ и $\Sigma_{2}$ всех их решений:

$$
\begin{aligned}
& \Sigma_{1}=\mu^{\downarrow}+\operatorname{Ann}_{\Lambda^{(n)}} K, \\
& \Sigma_{2}=\lambda^{\downarrow}+\operatorname{Ann}_{\Lambda^{(n)}} L,
\end{aligned}
$$

где $\mu^{\downarrow}$ и $\lambda^{\downarrow}$ - некоторые фиксированные решения соответственно систем уравнений (11) и (12),

$$
\begin{aligned}
K & =\left\langle\vec{A}_{1}, \ldots, \vec{A}_{k}\right\rangle, \\
L & =\left\langle\vec{A}_{1}, \vec{A}_{k+1}\right\rangle .
\end{aligned}
$$

Покажем, что

$$
\Sigma_{1} \cap \Sigma_{2} \neq \varnothing
$$

Пусть

$$
\vec{V} \in K \cap L
$$

то есть

$$
\begin{aligned}
\vec{V} & =\sum_{i=1}^{k} c_{i} \vec{A}_{i} \\
& =d_{1} \vec{A}_{1}+d_{k+1} \vec{A}_{k+1},
\end{aligned}
$$

где $c_{i}, d_{i} \in R$. Тогда справедливо равенство

$$
\left(c_{1}-d_{1}\right) \vec{A}_{1}+\sum_{i=2}^{k} c_{i} \vec{A}_{i}+\left(-d_{k+1}\right) \vec{A}_{k+1}=\overrightarrow{0} .
$$

Ввиду (13) вектор $\beta^{\downarrow}=\left(\beta_{1}, \ldots, \beta_{k+1}\right)^{T}$ можно записать в виде

$$
\beta^{\downarrow}=\left(\vec{A}_{1} \lambda^{\downarrow}, \vec{A}_{2} \mu^{\downarrow}, \ldots, \vec{A}_{k} \mu^{\downarrow}, \vec{A}_{k+1} \lambda^{\downarrow}\right)^{T} .
$$


Поэтому из (14) и $l c$-согласованности системы уравнений (1) выводим соотношение

$$
\left(c_{1}-d_{1}\right) \vec{A}_{1} \lambda^{\downarrow}+\sum_{i=2}^{k} c_{i} \vec{A}_{i} \mu^{\downarrow}+\left(-d_{k+1}\right) \vec{A}_{k+1} \lambda^{\downarrow}=\theta .
$$

Вычитая из равенства (15) почленно равенство (14), умноженное справа на $\lambda^{\downarrow}$, получаем равенство

$$
\sum_{i=2}^{k} c_{i} \vec{A}_{i}\left(\mu^{\downarrow}-\lambda^{\downarrow}\right)=\theta
$$

Так как

$$
\vec{A}_{1} \mu^{\downarrow}=\beta_{1}=\vec{A}_{1} \lambda^{\downarrow}
$$

из (16) следует равенство

$$
\sum_{i=1}^{k} c_{i} \vec{A}_{i}\left(\mu^{\downarrow}-\lambda^{\downarrow}\right)=\theta,
$$

означающее, что справедливо соотношение

$$
\mu^{\downarrow}-\lambda^{\downarrow} \in \operatorname{Ann}_{\Lambda^{(n)}} \vec{V}
$$

Таким образом, доказано включение

$$
\mu^{\downarrow}-\lambda^{\downarrow} \in \operatorname{Ann}_{\Lambda^{(n)}}(K \cap L) .
$$

Поскольку $\Lambda^{(n)}-\delta$-модуль, из (17) выводим соотношение

$$
\mu^{\downarrow}-\lambda^{\downarrow} \in \operatorname{Ann}_{\Lambda^{(n)}} K+\operatorname{Ann}_{\Lambda^{(n)}} L .
$$

Значит, существуют такие векторы $\gamma_{1}^{\downarrow} \in \operatorname{Ann}_{\Lambda^{(n)}} K$ и $\gamma_{2}^{\downarrow} \in \operatorname{Ann}_{\Lambda^{(n)}} L$, что

$$
\mu^{\downarrow}+\gamma_{1}^{\downarrow}=\lambda^{\downarrow}+\gamma_{2}^{\downarrow} \in \Sigma_{1} \cap \Sigma_{2}
$$

Следовательно, система уравнений (1) при $m=k+1$ разрешима.

Теорема 4 показывает, что всякий $\alpha$ - и $\delta$-модуль ${ }_{R} \Lambda$ является $l c$-разрешимым. Ввиду теоремы 2 можем указать подкласс класса $\mathscr{K}(R)$.

Теорема 5. Класс левых $R$-модулей, являюшихся $\alpha$ - $и \delta$-модулями, есть подкласс класса $\mathscr{H}(R)$ локально замкнутых справа $R$-модулей.

Применим полученные результаты в случае, когда $\Lambda=R$.

Определение 7. Кольцо $R$ назовем $\alpha$-кольцом ( $\delta$-кольцом), если модуль $R$ является $\alpha$-модулем ( $\delta$-модулем).

Из теоремы 5 получаем, что справедлива следующая теорема.

Теорема 6. Класс всех колеч, являюшихся $\alpha$ - и $\delta$-кольчами, есть подкласс класса всех локально замкнутых справа колеч. 
Укажем подкласс класса всех $\alpha$ - и $\delta$-колец.

Определение 8. Артиново справа и слева кольцо $R$ называется квазифробениусовым (QF-кольцом), если для любого его правого идеала $J$ и любого левого идеала $I$ справедливы равенства

$$
\begin{aligned}
& \mathrm{Ann}_{r} \mathrm{Ann}_{l} J=J, \\
& \mathrm{Ann}_{l} \mathrm{Ann}_{r} I=I,
\end{aligned}
$$

где для $S \subset R$ через $\mathrm{Ann}_{r} S\left(\mathrm{Ann}_{l} S\right)$ обозначен правый (левый) аннулятор $S$ в $R$ (см. стр. 327 в [4]).

Предложение $6([1,8])$. Если $R-Q F$-кольчо, то для любого $n \in \mathbf{N}$ и любых конечно порожденных подмодулей $F$ из $R_{R}^{(n)}$ и $G$ из $R R(n)$ справедливы равенства

$$
\begin{aligned}
& \operatorname{Ann}_{R^{(n)}} \operatorname{Ann}_{R(n)} F=F, \\
& \operatorname{Ann}_{R(n)} \operatorname{Ann}_{R^{(n)}} G=G
\end{aligned}
$$

Предложение 7. $Q F$-кольчо $R$ является $\alpha$ - и $\beta$-кольчом.

Доказательство. Ввиду первого из равенств (18) кольцо $R$ является $\alpha$-кольцом.

Пусть $K$ и $L-$ конечно порожденные подмодули из $R R(n), n \in \mathbf{N}$. Введем обозначения

$$
\begin{aligned}
& J_{1}=\operatorname{Ann}_{R^{(n)}} K, \\
& J_{2}=\operatorname{Ann}_{R^{(n)}} L .
\end{aligned}
$$

Ясно, что $J_{1}, J_{2}$ - подмодули из $R_{R}^{(n)}$. По утверждению 6 верны равенства

$$
\begin{aligned}
& \operatorname{Ann}_{R(n)} J_{1}=K, \\
& \operatorname{Ann}_{R(n)} J_{2}=L .
\end{aligned}
$$

Ввиду того, что

$$
\operatorname{Ann}_{R(n)} J_{1} \cap \operatorname{Ann}_{R(n)} J_{2}=\operatorname{Ann}_{R(n)}\left(J_{1}+J_{2}\right),
$$

справедливо равенство

$$
K \cap L=\operatorname{Ann}_{R(n)}\left(J_{1}+J_{2}\right)
$$

Отсюда получаем, что

$$
\begin{aligned}
\operatorname{Ann}_{R^{(n)}}(K \cap L) & =\operatorname{Ann}_{R^{(n)}} \operatorname{Ann}_{R(n)}\left(J_{1}+J_{2}\right)=J_{1}+J_{2} \\
& =\operatorname{Ann}_{R^{(n)}} K+\operatorname{Ann}_{R^{(n)}} L .
\end{aligned}
$$

Значит, $R-\delta$-кольцо.

В случае, когда $R$ - конечное коммутативное кольцо и $R \Lambda$ - конечный модуль, являющийся $(R, R)$-бимодулем со свойством $\mu r=r \mu$ для любых $\mu \in \Lambda$ и $r \in R$, предложение 7 доказано в [9].

Ввиду предложения 7 можем указать подкласс класса всех $\alpha$ - и $\delta$-колец.

Теорема 7. Класс всех $Q F$-колеч является подклассом класса всех $\alpha$ - $и \delta$-колеч. 


\section{Список литературы}

1. Елизаров В. П., Системы линейных уравнений над квазифробениусовыми кольцами. Фундамент. и прикл. матем. (1995) 1, №2, 535-539.

2. Елизаров В. П., Условия совместности систем линейных уравнений над кольцами. Фундамент. и прикл. матем. (2000) 6, №3, 777-788.

3. Елизаров В. П., Условия, необходимые для разрешимости системы линейных уравнений над кольцом. Дискретная математика (2004) 16, №2, 44-53.

4. Каш Ф., Модули и кольца. Мир, Москва, 1981.

5. Нечаев А. А., Линейные рекуррентные последовательности над квазифробениусовыми модулями. Успехи матем. наук (1993) 48, №3, 197-198.

6. Нечаев А. А., Конечные квазифробениусовы модули, приложения к кодам и линейным рекуррентам. Фундамент. и прикл. матем. (1995) 1, №1, 229-254.

7. Camion P., Levy L. S., Mann H. B., Linear equations over a commutative ring. J. Algebra (1971) 17, №3, 432-446.

8. Hall M., A type of algebraic closure. Ann. Math. (1939) 40, 360-369.

9. Nechaev A. A., Polylinear recurring sequences over modules and quasi-Frobenius modules. In: Proc. First Intern. Tainan-Moscow Algebra Workshop. de Gruyter, Berlin, 1996, pp. 283-288.

10. van der Waerden B. L., Moderne Algebra, v. 2. Springer, Berlin, 1931.

Статья поступила 17.01.2005. 